6-1-1995

\title{
A Stochastic Theory of Inhomogeneously Broadened Linewidths in Solids
}

Ulrich Zürcher

Cleveland State University, u.zurcher@csuohio.edu

Follow this and additional works at: https://engagedscholarship.csuohio.edu/sciphysics_facpub

Part of the Physics Commons

How does access to this work benefit you? Let us know!

Publisher's Statement

(C) 1995 American Institute of Physics.

\section{Repository Citation}

Zürcher, Ulrich, "A Stochastic Theory of Inhomogeneously Broadened Linewidths in Solids" (1995).

Physics Faculty Publications. 279.

https://engagedscholarship.csuohio.edu/sciphysics_facpub/279

This Article is brought to you for free and open access by the Physics Department at EngagedScholarship@CSU. It has been accepted for inclusion in Physics Faculty Publications by an authorized administrator of

EngagedScholarship@CSU. For more information, please contact library.es@csuohio.edu. 


\title{
A stochastic theory of inhomogeneously broadened linewidths in solids
}

\author{
Ulrich Zürchera) \\ Department of Chemistry, University of Kansas, Lawrence, Kansas 66045
}

(Received 27 April 1995; accepted 20 June 1995)

\begin{abstract}
We investigate spectral diffusion decay using a model for solids that consists of two-level-systems (TLSs) interacting via strain fields. For the case when the rate of TLS flips vanishes, we find algebraic decay of correlation functions of the local field. We show that properties of equilibrium fluctuations are in agreement with the hierarchical picture proposed by Basché and Moerner: TLSs far away produce fast fluctuations that are small in magnitude, and close TLSs produce large fluctuations that are less frequent. (c) 1995 American Institute of Physics.
\end{abstract}

\section{INTRODUCTION}

High-resolution spectroscopy of single molecules in solids has attracted considerable interest in recent years. ${ }^{1}$ This technique avoids the usual ensemble averaging that occurs when a large number of molecules are probed at the same time, making detailed tests of microscopic theories possible. Indeed, Basché and Moerner measured frequency shifts of perylene in polyethylene. They proposed an hierarchical picture of fluctuations of the "nano-environment;" impurities far away producing fast fluctuations that are small in magnitude, and close impurities producing large fluctuations that are less frequent. ${ }^{2}$

Because the environment of each impurity is different, each spectral line is a superposition of Lorentzian profiles, and the line is inhomogeneously broadened. In a real material, there are both static and dynamic contributions to the overall lineshape. The static contribution defines the overall lineshape on a very large frequency scale and usually varies dramatically depending upon the handling of the material. For example, the inhomogenous linewidth of pentacene in $p$-terphenyl crystals is observed to vary from $40 \mathrm{GHz}$ to 700 $\mathrm{MHz}$ depending simply upon the care taken to make the sample. In this paper, we ignore this static contribution and instead concentrate on the dynamic contribution to the lineshape which is controlled by some stochastic process.

The shift of the absorption frequency (the so-called local field) of the impurity $k$ is given by an Ising-type interaction $H_{k}=\Sigma_{j \neq k} v_{k j} \sigma_{j}$, where $\sigma_{j}$ is the pseudo-spin variable and where $v_{k j}$ is the interaction between the impurities $k$ and $j$. Averaging over the pseudo-spin variables, Gaussian and Lorentzian distributions of the local field follow for solids with high and low densities of impurities, respectively. ${ }^{3}$ While this approach yields, in principle, exact expressions for profiles of inhomogeneously broadened lines, we do not gain insights into the stochastic process of the local field. Spectral diffusion is the stochastic process of fluctuations of the local field caused by random spin flips.

Because at all times, an individual spin is either in its up or down state, only average values of spin variables relax. ${ }^{4}$ We may consider the average of a single spin variable during a time interval of length $\Delta t$. For $\Delta t$ much larger than the time-scale of spin flips, $\Delta t \gg \tau_{0}$, the spin spends equal

\footnotetext{
${ }^{a)}$ E-mail: zurcher@stout.chem.ukans.edu
}

times in its up and down state, and the average spin variable vanishes. For shorter time intervals, $\Delta t \sim \tau_{0}$, the spin spends more time in the state in which it was initially prepared. Taking the average over time intervals of variable length $t$, we find exponential decay of the spin variable, $\left\langle\sigma_{i}(t)\right\rangle_{t}=\exp \left(-t / \tau_{0}\right) \sigma_{i}(0)$. Alternatively, we may consider $N$ copies of the spin $\sigma_{i}$ and take the corresponding ensemble average. We find again exponential decay of the average spin, $\left\langle\sigma_{i}(t)\right\rangle_{N}=\exp \left(-t / \tau_{0}\right) \sigma_{i}(0)$. Correlation functions are then obtained by taking the average over the spin variable at the time $t=0,\left\langle\sigma_{i}(t) \sigma_{i}(0)\right\rangle_{t, N}=\exp \left(-t / \tau_{0}\right)\left\langle\sigma_{i}^{2}(0)\right\rangle$.

The local field is the (weighted) sum of an infinite sum of spins. Defining spectral diffusion, Klauder and Anderson made a particular choice and considered the case when the average over individual spins is taken first and then the behavior for an infinite number of spins is studied. ${ }^{5}$ Most work on spectral diffusion follows this approach. Recently, Zumofen and Klafter studied spectral diffusion for a large but finite number of spins. ${ }^{6}$ They observed that the flip of any spin may cause a change in the local field. Thus, the characteristic time-scale of fluctuations of the local field is inversely proportional to the number of spins. For a large number of spins, the time-scale of fluctuations of the local field is much shorter than that of a single spin flip. Furthermore, a particular configuration with a majority of spins in either the up or down state occurs with vanishing probability. Spin flips cause an initial configuration with an unequal number of spins in the up and down state to relax towards the stable equilibrium configuration.

In this paper, we use the approach proposed by Zumofen and Klafter to investigate the spectral diffusion of an infinite number of spins. We derive properties of the local field that are in agreement with the hierarchical picture of fluctuations proposed by Basché and Moerner.

The outline of this paper is as follows. In Sec. II, we review briefly the theory of spectral diffusion by Klauder and Anderson and discuss the crossover from Lorentzian to Gaussian diffusion in their model. In Sec. III, we derive the hierarchical picture of fluctuations of the local field for times shorter than the characteristic time-scale of individual spin flips. Properties of the local field in the stationary state are discussed in Sec. IV. We find that line profiles are given by Lévy-distributions. The crossover form Lorentzian to Gaussian line shapes is analyzed in more detail in Sec. V. Finally, we summarize our results in Sec. VI. 


\section{SPECTRAL DIFFUSION: THEORY BY KLAUDER AND ANDERSON}

In solids, vibrations of ionic cores lead to elementary excitations that are extended throughout the sample (phonons). In many cases, additional degrees of freedom exist that are associated with, e.g., changes in local configurations. In a widely used model, two-level-systems (TLSs) are introduced by truncating the spectrum of these local modes to their ground and first excited states. ${ }^{7,8}$ The TLSs are coupled to phonons and the TLS energy-splitting is renormalized, $E_{k} \rightarrow E_{k}+H_{k}$. The local field is of dipolar form ${ }^{9}$ which we write for later use as

$$
H_{k}=\gamma \sum_{j \neq k} \frac{1}{r_{k j}^{\kappa}} \sigma_{j}
$$

Here, $r_{k j}$ is the distance between the TLSs $k$ and $j$, and $\sigma_{j}$ is the pseudo-spin variable, $\sigma_{j}= \pm \frac{1}{2}$. We consider values of the exponent $0 \leqslant \kappa \leqslant 3$ so that the local field decreases more slowly than the dipolar field $(\kappa=3)$.

We assume that the spin $j$ is initially in the state $\sigma_{j}(0)$ and jumps subsequently between its two levels with probability per unit time $\tau_{0}^{-1}$. Averaging over the realizations of the spin variable, we find its conditional probability,

$$
\mathscr{P}\left[\sigma_{j}, t ; \sigma_{j}(0), 0\right]=e^{-t / \tau_{0}} \delta_{\sigma_{j} \sigma_{j}(0)}+\left(1-e^{-t / \tau_{0}}\right) \mathscr{P}_{\text {eq }} .
$$

Here, $\mathscr{P}_{\text {eq }}$ is the equilibrium distribution,

$$
\mathscr{P}_{\text {eq }}=\frac{1}{2}(|\uparrow\rangle\langle\uparrow|+| \downarrow\rangle\langle\downarrow|) \text {. }
$$

Spectral diffusion is the conditional probability of the local field. Klauder and Anderson consider the case when the average over realizations of the spin-variables is taken first and thus define

$$
p\left(H, t ; H_{0}, 0\right)=\left\langle\delta\left(H-\gamma \sum_{j \neq k} \frac{\sigma_{j}}{r_{k j}^{\delta}}\right)\right\rangle .
$$

Here, $\langle\cdot\rangle$ denotes the average with respect to the conditional probability of individual spins $P\left[\sigma_{j}, t ; \sigma_{j}(0), 0\right]$. Using some approximations, one finds for short times $t \rightarrow 0$,

$$
\begin{aligned}
p\left(H, t ; H_{0}, 0\right)= & \frac{1}{2 \pi} \int_{-\infty}^{\infty} d y \exp \left[i y\left(H-H_{0}\right)\right. \\
& \left.-\tau_{0}^{-1} t K(y)\right],
\end{aligned}
$$

where $K(y)$ can be approximated by an integral,

$$
K(y)=4 \pi n \int_{r_{\min }}^{\infty} d r r^{2}\left(1-\cos \frac{\gamma y}{r^{\kappa}}\right) .
$$

Here, $n$ is the density of spins, and $r_{\min }$ is the distance to the nearest spin, $r_{\min } \rightarrow 0$. Depending on the value of $y$, we find

$$
K(y)=\left\{\begin{array}{l}
\left(2 \pi^{2} / 3\right) n \gamma|y|, \quad|y|>2 \pi r_{\min }^{\kappa} / \gamma, \\
(2 \pi / 3) n(y \gamma)^{2} / r_{\min }^{\kappa}, \quad y \rightarrow 0,
\end{array}\right.
$$

so that $K(y) \propto|y|$ except when $y$ is small, in which case $K(y) \propto y^{2}$. Thus, for a small difference $H-H_{0}$, the spectral diffusion is a Lorentzian whose width grows linearly with time,

$$
w_{L}(t)=\frac{2 \pi^{2} \gamma}{3} n \tau_{0}^{-1} t,
$$

while for $H-H_{0}$ large, the distribution is a Gaussian whose variance grows linearly with time,

$$
w_{G}(t)=\frac{2 \pi \gamma^{2}}{3 r_{\min }^{\delta}} n \tau_{0}^{-1} t
$$

The local field approaches a stationary state for long times. For this state, Klauder and Anderson find a Lorentzian distribution that goes over into a Gaussian at $H \sim H_{c r}$ $=(\gamma / 2 \pi) r_{\min }^{-\kappa}$. For two different values of the exponent, $\kappa_{1}<\kappa_{2}$, we have $H_{c r}\left(\kappa_{1}\right)<H_{c r}\left(\kappa_{2}\right)$ so that the profile is more like a Lorentzian for larger values of $\kappa$. We observe that a Lorentzian profile with Gaussian wings is not a stable distribution, ${ }^{10}$ from which we conclude that the theory by Klauder and Anderson is correct only in some approximate manner.

\section{RELAXATION OF THE LOCAL FIELD}

We consider the case when the rate of flips of a single spin is arbitrarily small, $\tau_{0}^{-1} \rightarrow 0$. It follows that during a finite time-interval, the probability for a particular spin flip to occur is vanishingly small. Because we consider a system with an infinite number of spins, however, changes in the local field occur with some finite probability during time intervals of non-zero lengths.

We observe that the flip of a close spin causes a larger change in the local field than the flip of a spin far away. We introduce concentric "shells" around the spin $\sigma_{k}$. For two spins $\sigma_{j}$ and $\sigma_{l}$ in the same shell, $\rho_{n}<r_{k j}, r_{k l}<\rho_{n+1}$, simultaneous flips, $\sigma_{j}=+1 \rightarrow \sigma_{j}=-1$ and $\sigma_{l}=-1 \rightarrow \sigma_{l}=+1$, cancel each other out and the local field does not change. We neglect cancellations of spin flips across different shells and thus write the local field as

$$
H=\gamma \sum_{n=1}^{\infty} \frac{N_{n}}{\rho_{n}^{\kappa}} s_{n} .
$$

Here, $N_{n}$ is the number of TLSs in the $n$-th shell and $s_{n}$ is the superposition of spins in that shell,

$$
s_{n}=\frac{1}{N_{n}} \sum_{\rho_{n}<r_{k j}<\rho_{n+1}} \sigma_{j} .
$$

We note that the value of $s_{n}$ is bounded, $-\frac{1}{2}<s_{n}<+\frac{1}{2}$.

Because each spin is in its ground and excited state with equal probability, the expectation value of the superposition of spins vanishes, $\left\langle s_{n}\right\rangle_{\mathrm{eq}}=0$, and its variance decreases as the number of spins increases. Using the equipartition theorem we have $\left(k_{B}=1\right)$,

$$
\left\langle s_{n}^{2}\right\rangle_{\mathrm{eq}}=\frac{T}{N_{n}} .
$$

Further, flipping any spin in the $n$-th shell may cause a change in $s_{n}$, and the characteristic time-scale of the process $s_{n}(t)$ is inversely proportional to $N_{n}$,

$$
\tau_{n}=\frac{\tau_{0}}{N_{n}} .
$$


An initial configuration of spins with $s_{n} \neq 0$ relaxes towards the equilibrium configuration $s_{n}=0$ on the time-scale $\tau_{n}$, $s_{n}(t)=\exp \left(-t / \tau_{n}\right) s_{n}(0)$. The correlation function follows as $\left\langle s_{n}(t) s_{n}(0)\right\rangle_{0}=\exp \left(-t / \tau_{n}\right)\left\langle s_{n}^{2}\right\rangle_{0}$. We assume thermal equilibrium at $t=0$ so that $\left\langle s_{n}^{2}\right\rangle_{0}=\left\langle s_{n}^{2}\right\rangle_{\mathrm{eq}}$. It follows

$$
\left\langle s_{n}(t) s_{n}(0)\right\rangle_{0}=\frac{T}{N_{n}} \exp \left(-\frac{N_{n} t}{\tau_{0}}\right) .
$$

The relaxation of the local field is induced by the relaxation of the superposition of spins, Eq. (10),

$$
H(t)=\gamma \sum_{n=1}^{\infty} \frac{N_{n}}{\rho_{n}^{\kappa}} s_{n}(t) .
$$

We assume that the number of spins is different in each shell, so that the stochastic processes $s_{n}(t)$ and $s_{m}(t)$ with $n$ $\neq m$ have different characteristic time-scales. It follows that correlations between the two processes can be neglected, $\left\langle s_{n}(t) s_{m}(0)\right\rangle_{\mathrm{eq}}=\delta_{n m} \exp \left(-N_{n} t / \tau_{0}\right)\left\langle s_{n}^{2}\right\rangle_{\mathrm{eq}}$. For equilibrium correlations of the local field we find

$$
\langle H(t) H(0)\rangle_{\mathrm{eq}}=\gamma^{2} T \sum_{n=1}^{\infty} \frac{N_{n}}{\rho_{n}^{2 \kappa}} e^{-N_{n} t / \tau_{0}} .
$$

Thus, we recover the hierarchical picture proposed by Basché and Moerner for fluctuations of the local field: Spins far away produce fast fluctuations that are small in magnitude while close spins produce slow fluctuations that are large in magnitude. It follows in particular that the relaxation of the local field is characterized by a broad distribution of time-scales.

For shells of constant thickness, $\Delta \rho=\rho_{n+1}-\rho_{n}$ $=$ const, we assume an homogeneous distribution of spins. The number of spins is proportional to the volume of the shell,

$$
N_{n}=N_{0} \rho_{n}^{2},
$$

where $N_{0}$ is proportional to the density of spins, $N_{0} \propto n$. We insert Eq. (17) into Eq. (16), $\langle H(t) H(0)\rangle_{\text {eq }}$ $=\gamma^{2} T \Sigma_{n} N_{0} \rho_{n}^{2-2 \kappa} \exp \left(-N_{0} \tau_{0}^{-1} \rho_{n}^{2} t\right)$. For $\Delta \rho \rightarrow 0$, the series goes over into an integral

$$
\begin{aligned}
\langle H(t) H(0)\rangle_{\mathrm{eq}}= & 4 \pi^{2} \gamma^{2} T N_{0} \int_{0}^{\infty} d \rho \rho^{4-2 \kappa} \\
& \times \exp \left(-N_{0} \tau_{0}^{-1} \rho^{2} t\right) .
\end{aligned}
$$

We separate the time-dependent part of the RHS of Eq. (18) and find

$$
\langle H(t) H(0)\rangle_{\mathrm{eq}}=2 \int_{0}^{\infty} I(u) K(u, t) d u .
$$

Here, $K(u, t)$ is the Green's function of the one-dimensional diffusion equation,

$$
K(u, t)=\frac{1}{\sqrt{4 N_{0} \tau_{0}^{-1} t}} \exp \left(-\frac{u^{2}}{4 N_{0} \tau_{0}^{-1} t}\right),
$$

and the function $I(u)$ is given by,

$$
I(u)=4 \pi^{2} \gamma^{2} T N_{0} \int_{0}^{\infty} d \rho \rho^{4-2 \kappa} \cos (u \rho) .
$$

For $\kappa=2$ and $\kappa=3, I(u)$ is singular at the origin $u=0$, $I(u, \kappa=2) \sim \delta(u)$ and $I(u, \kappa=3) \sim \delta^{\prime \prime}(u)$, and the decay of the correlation function depends only on the behavior of the diffusion kernel at the origin. Indeed, this property is true for arbitrary values of $\kappa$, and the correlation function of the local field is proportional to the return probability of a (symmetric) random walker in $D=7-2 \kappa$ spatial dimensions. For $\kappa=3$ we have $D=1$. Since a one-dimensional random walker returns to its origin with probability one, ${ }^{11}$ we infer

$$
\langle H(t) H(0)\rangle_{\mathrm{eq}} \rightarrow \text { const, } \quad t \rightarrow \infty \quad \text { for } \kappa=3 .
$$

For $\kappa \leqslant 2$, we find algebraic decay of the correlation function,

$$
\langle H(t) H(0)\rangle_{\mathrm{eq}} \sim \frac{1}{t^{5 / 2-\kappa}}, \quad t \rightarrow \infty \quad \text { for } \kappa \leqslant 2 .
$$

We note that for $\kappa=2$ and $\kappa=3$ correlations of the local field are persistent as they do not define a finite "effective" relaxation time, $\int_{0}^{\infty}\langle H(t) H(0)\rangle_{\mathrm{eq}} d t \propto \tau_{\text {eff }} \rightarrow \infty$ for $\kappa=2,3$.

\section{EQUILIBRIUM FLUCTUATIONS}

At non-zero temperatures, relaxation is accompanied by thermal fluctuations. This is the central statement of the fluctuation-dissipation theorem (FDT) (Ref. 12) which in the case of Brownian motion relates the diffusion constant to the damping constant. Because the correlation function of the superposition of spins decays exponentially in time, Doob's theorem holds and thermal fluctuations of $s_{n}(t)$ are described by a Gauss-Markov process. ${ }^{13}$ Thus, $s_{n}(t)$ spreads diffusively in time,

$$
\left\langle\left[s_{n}(t)-s_{n}(0)\right]\right\rangle_{\mathrm{eq}}=D_{n} t .
$$

From Eqs. (12) and (13) we find that the diffusion constant is independent of the number of spins,

$$
D_{n}=\frac{\left\langle s_{n}^{2}\right\rangle_{\mathrm{eq}}}{\tau_{n}}=\frac{T}{\tau_{0}}=\text { const. }
$$

Furthermore, the stationary distribution of $s_{n}$ is a Gaussian with variance $\left\langle s_{n}^{2}\right\rangle_{\mathrm{eq}}=T N_{n}^{-1}$,

$$
p_{s t}\left(s_{n}\right)=\sqrt{\frac{1}{\pi T N_{n}^{-1}}} \exp \left(-\frac{s_{n}^{2}}{T N_{n}^{-1}}\right) .
$$

The noise spectrum of the local field is defined by $S_{H}(\omega)=(1 / 2 \pi) \int_{-\infty}^{\infty}\langle H(t) H(0)\rangle_{\mathrm{eq}} \exp (i \omega t) d t$. From Eqs. (22) and (23), we find in the limit $\omega \rightarrow 0$,

$$
S(\omega) \sim \begin{cases}1 / \omega, & \kappa=3, \\ 1 / \sqrt{\omega}, & \kappa=2, \\ \text { const, } & \kappa=1,0 .\end{cases}
$$

That is, the noise spectrum is "white" for $\kappa=0$ and $\kappa=1$, while it is "colored" for $\kappa=2$ and $\kappa=3$.

In thermal equilibrium, the time evolution of the local field is governed by a stochastic "force,"

$$
H(t)=\int_{0}^{t} \eta(s) d s .
$$

For white noise the stochastic force is Markovian, $\langle\eta(t) \eta(s)\rangle \sim \delta(t-s)$, while for colored noise long-time cor- 
relations exist. We invoke Onsager's regression hypothesis which states that correlations of fluctuating variables at times $t$ and 0 in equilibrium systems are the same as averages of fluctuating variables at time $t$, given that certain fluctuations have occured at time $0 .{ }^{12}$ We find for large $t-s$, $\langle\eta(t) \eta(s)\rangle_{\mathrm{eq}} \sim\langle H(t) H(s)\rangle_{\mathrm{eq}}$ so that

$$
\langle\eta(t) \eta(s)\rangle_{\mathrm{eq}} \sim \begin{cases}(t-s)^{0}, & \kappa=3, \\ (t-s)^{-1 / 2}, & \kappa=2, \\ \delta(t-s), & \kappa=1,0 .\end{cases}
$$

Using Eq. (28), the mean square displacement of the local field follows as $m(t)=\left\langle[H(t)-H(0)]^{2}\right\rangle_{\mathrm{eq}}$ $=2 \int_{0}^{t} d s \int_{0}^{s} d s^{\prime}\left\langle\eta(s) \eta\left(s^{\prime}\right)\right\rangle_{\mathrm{eq}}$. From Eq. (29), we find diffusive and superdiffusive behavior for long times,

$$
m(t) \sim \begin{cases}t^{2}, & \kappa=3, \\ t^{3 / 2}, & \kappa=2, \\ t, & \kappa=1,0 .\end{cases}
$$

The Hurst exponent $h$ is defined by $m(t) \sim t^{2 h}$ with $0 \leqslant h$ $\leqslant 1$ so that $h=\frac{1}{2}$ corresponds to ordinary Brownian diffusion. We have $h=1$ for $\kappa=3, h=\frac{3}{4}$ for $\kappa=2$, and $h=\frac{1}{2}$ for $\kappa=1,0$.

Super-diffusive behavior may result from a stochastic process $x(t)$ that is stationary and has Gaussian increments. ${ }^{10}$ For long times, the stochastic process approaches a unique stationary state in which the distribution of the fluctuating variable $x$ is given by $p_{d}(x)=\pi^{-1} \int_{0}^{\infty} \exp \left(-u^{d}\right) \cos (u x) d u$ with $1 \leqslant d \leqslant 2$. These are the Lévy-distributions and the parameter $d$ is related to the Hurst exponent by $d=1 / h$. For ordinary Brownian motion we have $h=\frac{1}{2}$ and hence $d=2$, so that we recover the Gaussian property of its stationary distribution. Super-diffusive behavior with $h=1$ yields $d=1$ and this Lévy-distribution is a Lorentzian.

From Eq. (30) we find the parameter characterizing the stationary distribution of the local field. We have $d=1$ for $\kappa=3$ and the distribution is a Lorentzian,

$$
p_{s t}(H)=\frac{1}{\pi} \frac{\lambda}{H^{2}+\lambda^{2}}, \quad \kappa=3,
$$

where $\lambda$ is the width of the Lorentzian profile. As $\kappa$ decreases, the distribution becomes more narrow at the origin. A Gaussian distribution follows for $\kappa=1,0$,

$$
p_{s t}(H)=\frac{1}{\sqrt{\pi \mu}} \exp \left(-\frac{H^{2}}{\mu}\right), \quad \kappa=1,0,
$$

where $\mu$ is the variance of the Gaussian profile. For $\kappa=2$, the line profile lies between a Gaussian and a Lorentzian. In Sec. II, we found a similar dependence of the line profile on the interaction between spins. If the interaction decays more slowly than the dipolar field, fluctuations of the local field are dominated by flips of spins far away. The variance of these fluctuations are small, and the Gaussian line profile is a consequence of the central limit theorem. ${ }^{11}$

\section{DISCUSSION}

The theory of inhomogeneously broadened lineshapes has been re-investigated by Kador. ${ }^{14} \mathrm{He}$ considers the situa- tion in which point defects are excluded from a spherical region of radius $R_{c}$ around the impurity. Two limiting cases are found depending on the density of defects. For $\rho R_{c}^{3}$ $\ll 1$, a Lorentzian profile is obtained, while for $\rho R_{c}^{3} \gg 1$, a Gaussian profile follows. In the latter case, the impurity is in the center of an empty space and a large number of defects is already present in the first coordination shell. The relative size of fluctuations is small for a large number of defects and the central limit theorem can be applied to give a Gaussian profile. In the former case, the impurity does not "notice" the volume that is inaccessible to the defects.

This derivation of inhomogeneous lineshapes has been criticized by Skinner and co-workers. ${ }^{3}$ They point out that the inequality $\rho R_{c}^{3}$ can be satisfied in two different ways. For finite $R_{c}$, we let $\rho$ become large which contradicts the assumption of low defect densities implicit in continuum theories. Alternatively, for fixed (and small) $\rho$, we let $R_{c}$ become large. In this case, it is not clear physically why defects should be excluded from a large sphere around the impurity. They propose a scheme in which nearby, strongly interacting defects are treated in an exact manner, while defects farther away are treated in an approximative manner. Gaussian and Lorentzian profiles are recovered in the limit of large and small densities of defects, respectively.

We expect that the central limit theorem applies to any inhomogeneous lineshape. Lévy generalized the standard derivation of the Gaussian distribution and introduced socalled stable distributions. ${ }^{10}$ These are the Lévy-distributions with Gaussian and Lorentzian distributions as limiting cases. For non-Gaussian Lévy-distributions, moments of the random variable do not exist for arbitrarily high orders, which implies scale-invariant properties. For the Gaussian distribution, a scale is defined by the second moment. In the above mentioned continuum theories, scale-invariance is destroyed by assuming that the impurity is surrounded by an empty space. It follows that a large number of defects, $N_{1}$, is located in the first coordination shell. In our stochastic theory, this assumption defines the slowest "mode" $s_{1}(t)$ with $\left\langle s_{1}(t) s_{1}(0)\right\rangle_{\mathrm{eq}}=\exp \left(-N_{1} \tau_{0}^{-1} t\right)\left\langle s_{1}^{2}\right\rangle_{\mathrm{eq}}$. This mode determines the long-time behavior of the local field, $\langle H(t) H(0)\rangle_{\mathrm{eq}}$ $\simeq \exp \left(-N_{1} \tau_{0}^{-1} t\right)\left\langle H^{2}(0)\right\rangle_{\mathrm{eq}}$, and diffusive behavior follows, $\left\langle[H(t)-H(0)]^{2}\right\rangle_{\mathrm{eq}} \sim t$ for $t \gg \tau_{0} / N_{1}$. The corresponding stationary distribution of the local field is a Gaussian.

Spectral diffusion decay of individual pentacene molecules in $p$-terphenyl crystals is studied in recent papers by Reilly and Skinner. ${ }^{15}$ They take first the average over individual spins and then consider the superposition of spins. For a slow rate of spin flips, they arrive at an expression for the spectral diffusion with adjustable parameters. The values of these parameters are determined at some (lower) temperature so that the theory does not contain free parameters at a (higher) temperature. At the higher temperature, this theory gives a probability for small changes of the local field that is smaller than experimental values obtained by analyzing individual trajectories of the local field. It is remarkable that for both the lower and higher temperature, the experimental data is in close agreement with predictions of Lorentzian diffusion. 


\section{SUMMARY}

We have outlined a stochastic theory of inhomogeneously broadened linewidths in solids that is in qualitative agreement with the hierarchical picture for fluctuations of the local field proposed by Basché and Moerner. Spins far away produce fast fluctuations that are small in magnitude while close spins produce large fluctuations that are infrequent. We have derived algebraic decay of time-correlations of the local field. The line profile is given by the stationary probability density of the local field. Depending on the decay of the interaction between spins, we have found Lévy-distributions from which Gaussian and Lorentzian profiles follow as limiting cases.

We have developed a theory of spectral diffusion decay by first considering finite superpositions of spins. In the most probable configuration, half of the spins are in the up- and down-state. An initial spin configuration, with an unequal occupation of spin-states, approaches this stationary configuration for long times. Such probabilistic concepts enter the thermodynamic description of an infinite system via the entropy term of the free energy. We are not the first to consider entropy to derive slow relaxational properties. Indeed, the relevance of entropy has been discussed for enhanced diffusion in one-dimensional maps ${ }^{16}$ and the non-Arrhenius behavior of the viscosity and diffusion constant in super-cooled liquids. ${ }^{17}$

\section{ACKNOWLEDGMENTS}

The research at the University of Kansas is supported by the Petroleum Research Fund under Grant No. 27847-G5. I would like to thank Professor B. B. Laird for providing a stimulating research environment and Professor R. J. Silbey for his hospitality at M.I.T. where this project was initiated.

${ }^{1}$ W. E. Moerner, Science 265, 46 (1994)

${ }^{2}$ Th. Basché and W. E. Moerner, Nature 355, 335 (1992).

${ }^{3}$ D. L. Orth, R. J. Mashl, and J. L. Skinner, J. Phys.: Conden. Matter 5, 2533 (1993).

${ }^{4}$ It is perhaps helpful to recall the situation for a damped free particle. The deterministic relaxation of its coordinate $x(t)=x(0) \exp (-\gamma t)$ is modified by thermal fluctuations. The combined effect of relaxation and fluctuations is described by the Langevin equation, $\dot{x}(t)=-\gamma x(t)+\xi(t)$. Because the noise is additive, $\langle x(t) \xi(s)\rangle=0$, we have $\langle x(t) x(0)\rangle=\left\langle x^{2}(0)\right\rangle \exp (-\gamma t)$. The average on the RHS is taken with respect to the coordinate at the initial time $t=0$.

${ }^{5}$ J. R. Klauder and P. W. Anderson, Phys. Rev. 125, 912 (1962).

${ }^{6}$ G. Zumofen and J. Klafter, Chem. Phys. Lett. 219, 303 (1994).

${ }^{7}$ P. W. Anderson, B. I. Halperin, and C. M. Varma, Philos. Mag. 25, 1 (1972).

${ }^{8}$ W. A. Phillips, J. Low Temp. Phys. 7, 351 (1972).

${ }^{9}$ J. Joffrin and A. Levelut, J. Phys. (Paris) 36, 811 (1972).

${ }^{10}$ B. B. Mandelbrot, The Fractal Geometry of Nature (Freeman, San Francisco, 1982).

${ }^{11}$ W. Feller, An Introduction to Probability Theory and its Applications, 2nd ed. (Wiley, New York, 1957), Vol. I.

${ }^{12}$ See, e.g., L. E. Reichl, A Modern Course in Statistical Physics (University of Texas, Austin, 1987).

${ }^{13}$ M. C. Wang and G. E. Uhlenbeck, Rev. Mod. Phys. 17, 323 (1945); reprinted in Selected Papers on Noise and Stochastic Processes, edited by N. Wax (Dover, New York, 1954).

${ }^{14}$ L. Kador, J. Chem. Phys. 95, 5574 (1991).

${ }^{15}$ P. D. Reilly and J. L. Skinner, Phys. Rev. Lett. 71, 4257 (1993); J. Chem. Phys. 101, 959, 965 (1994); 102, 1540 (1995).

${ }^{16}$ R. Stoop, Europhys. Lett. 29, 433 (1995).

${ }^{17}$ U. Mohanty, I. Oppenheim, and C. H. Taubes, Science 266, 425 (1994). 
The Journal of Chemical Physics is copyrighted by the American Institute of Physics (AIP). Redistribution of journal material is subject to the AIP online journal license and/or AIP copyright. For more information, see http:/ojps.aip.org/jcpo/jcpcr/jsp Copyright of Journal of Chemical Physics is the property of American Institute of Physics and its content may not be copied or emailed to multiple sites or posted to a listserv without the copyright holder's express written permission. However, users may print, download, or email articles for individual use. 
The Journal of Chemical Physics is copyrighted by the American Institute of Physics (AIP). Redistribution of journal material is subject to the AIP online journal license and/or AIP copyright. For more information, see http://ojps.aip.org/jcpo/jcper/jsp 